\title{
A new breed of computer users: Rats control a cursor via joystick manipulation
}

\author{
DAVID A. WASHBURN \\ Georgia State University, Atlanta, Georgia \\ MICHAEL J. RULON \\ Covenant College, Lookout Mountain, Tennessee \\ and \\ JONATHAN P. GULLEDGE \\ Lee University, Cleveland, Tennessee
}

\begin{abstract}
Many exciting findings have been reported in the 15 years since monkeys were first demonstrated to have the capacity to perform computerized tasks. The present data indicate that albino rats can also learn to respond to computer-generated stimuli by manipulating a joystick. Although the rat's control of the cursor is not as skillful as has been reported for primate species, it is clearly better than chance and suggests the great potential for comparative investigation afforded by use of the computer test system.
\end{abstract}

Almost 15 years ago, it was reported that rhesus monkeys (Macaca mulatta) could learn to respond to computergenerated stimuli by manipulating a joystick, which in turn controlled the movements of a cursor on the computer screen (Rumbaugh, Richardson, Washburn, SavageRumbaugh, \& Hopkins, 1989). Since that time, the computerized test system has been used successfully in many experiments, at many laboratories, and with a variety of primate species. The test system provides a rich array of challenges, yields thousands of responses per day from each animal, affords control to the animals over what they work on and when they work, and supports the psychological well-being of primates maintained for research purposes (e.g., Andrews, 1993; Vauclair, Fagot, \& Hopkins, 1993; Washburn \& Rumbaugh, 1992b). Perhaps for these reasons, the data collected with this apparatus have overturned some of the long-held beliefs about the competencies of rhesus monkeys, revealing the species to be capable of producing many empirical phenomena previously demonstrated only with humans and apes. For example, monkeys have been shown to be capable of resolving situations involving stimulus-response spatial discontiguity, to manifest relational as well as associative learning, to

This research was supported by grants from the National Institutes of Health (HD-38051) and from the National Aeronautics and Space Administration (NAG2-1271). Additional support was provided by Covenant College and by the College of Arts and Sciences of Georgia State University. Correspondence concerning this article should be addressed to D. A. Washburn, Department of Psychology, Georgia State University, Atlanta, GA 30303 (e-mail: dwashburn@gsu.edu). benefit in performance from perceived control and competition, to have performance disrupted by Stroop-like interference and by social isolation, to represent the invisible movements of displaced stimuli, to respond adaptively to subjective uncertainty, and to respond as predictoroperators on tests of psychomotor function (Filion, Washburn, \& Gulledge, 1996; Smith, Shields, Schull, \& Washburn, 1997; Washburn, Hopkins, \& Rumbaugh, 1990; Washburn \& Rumbaugh, 1991a, 1991b, 1992a).

Because the test system has proven to be so valuable for studies of human and nonhuman primate behavior, it would be desirable to find other animals that can also use the apparatus. In particular, we were interested in determining whether rodents could master the joystick manipulation skills required to respond to computerized tasks. Previous work in which rats have used a "joystick" (e.g., Heyes \& Dawson, 1990; Mitchell, Heyes, Gardner, \& Dawson, 1999; Ray, Gardner, \& Heyes, 2000) required only that the animals move the lever bidirectionally, and not to use it to control the movements of computer-generated stimuli. One could, of course, use a different manipulandum, such as a touchscreen, to permit the animals to respond to computer graphic stimuli. Rats have been successfully trained to perform two-choice discrimination-learning tasks by touching stimuli on a screen (Astur et al., 1996; Bussey, Dias, Amin, Muir, \& Aggleton, 2001; Markham, Butt, \& Dougher, 1996; Matzke, 1999). Touchscreens are much more expensive than joysticks, however, and can be poorly suited to motor tasks, such as pursuit tracking. In addition, the training on the remote cause-effect relations inherent in the use of a joystick to control a cursor may produce gen- 
eralized effects on the capacity for relational learning. This reasonable hypothesis, which has yet to be tested, presumes a benefit of joystick-based training that would not be obtained in touchscreen paradigms in which animals respond directly to computer-generated stimuli.

Rats and other rodents remain popular species for biomedical research. Although their popularity in psychological and pedagogical research appears to have waned in recent decades, they do still provide a common animal model for studies of learning, memory, and psychopharmacology. Numerous benefits might be realized if these studies could utilize the exact joystick-based tasks that are employed with humans, apes, and monkeys. Direct comparison of performance (and of the variables that influence performance) across animals with vastly different brains and developmental histories would be particularly useful for understanding the relations between the behavioral, neural, chemical, and other biological levels of analysis. However, it seemed quite possible that rodents would lack the visual acuity, manual strength and dexterity, and cognitive capacity to bridge the stimulus-response spatial discontiguity and to learn the cause-effect relationships necessary to manipulate a joystick skillfully.

\section{EXPERIMENT 1}

\section{Method}

Subject. One adult female albino rat was tested in this study. The animal (weight $=240 \mathrm{~g}$ ) had received traditional operant leverpressing training prior to the present study. Food was restricted to maintain the rat at between $90 \%$ and $100 \%$ of its free-feeding weight, after which it was able to provide ample food for itself to remain at its free-feeding weight. The rat was trained and tested in its home cage, with water continuously available.

Apparatus. A stainless steel rodent cage $(23 \mathrm{~cm}$ wide $\times 38 \mathrm{~cm}$ long $\times 23 \mathrm{~cm}$ high) was modified for this study. The $23 \times 23 \mathrm{~cm}$ mesh wall at the front of the cage was removed and replaced with transparent Lexan. A hole (4 cm in diameter) was cut in this Lexan wall, centered around a point approximately $6.35 \mathrm{~cm}$ from the floor of the cage and $11.5 \mathrm{~cm}$ from the left wall of the cage. A standard analog joystick (Kraft KC-3) was mounted to the Lexan so that the joystick handle protruded into the cage through this hole (see Figure 1). To keep the joystick base from blocking too much of the Lexan window, the joystick handle and electronics were removed from the base and buttons prior to mounting, so that only the potentiometers, cams, and wires hung on the outside of the cage. To make the joystick easier to manipulate, it was further modified by removing both of the springs that would otherwise return the stick to the center position. Also, the plastic handle was removed, exposing the metal shaft, which could be manipulated from within the cage. Given these modifications, only $20 \mathrm{~g}$ of load were required to move the joystick handle $(0.02 \mathrm{~N}$ of force).

The joystick was connected to the game board of a 16-MHz, 386based computer, which in turn was connected to a 13-in. VGA monitor. The monitor was positioned directly behind the joystick, and all the stimuli presented on this monitor were restricted to a $23 \times 14 \mathrm{~cm}$ area that was visible through the Lexan window of the cage. A pellet dispenser (Gerbrands 5120) was mounted atop the cage and was used to drop 97-mg chow pellets (P. J. Noyes, Lancaster, NH) through the top of the cage, down a copper tube, and into a metal cup mounted on the cage floor. The dispenser was connected to the computer with an interface board and relay box (Keithley-Metrabyte PIO-12 and ERA-01). An external speaker/amplifier was positioned directly below the joystick and connected to the computer, so that computergenerated auditory feedback would emanate from the general direction of the monitor.

Tasks. Except for the modifications to the joystick, this apparatus was identical to that used with rhesus monkeys (Washburn \& Rumbaugh, 1992c). Similarly, the tasks used to train the rat were comparable to those described for the training of rhesus monkeys (Rumbaugh et al., 1989). The first task (side) began with the cursor (a $1.25 \times 1.25 \mathrm{~cm}$ plus sign) in the middle of the screen, surrounded on all sides by blue rectangles (walls). A deflection of the joystick resulted in a proportional movement of the cursor on the screen. Note the difference between this method of cursor movement and that used in previous studies with rhesus monkeys (e.g., Rumbaugh et al., 1989). With a joystick that automatically centers when released (as has been used with monkeys), the cursor moves in a direction isomorphic to the angle of joystick displacement. However, the rats could not move the joystick handle with the springs attached. Without those springs, the cursor moved proportionally to the deflection of the joystick (i.e., if it was moved halfway between the center position and the rightmost position, the cursor moved halfway between the center and the rightmost position). Otherwise, the smallest movement of the joystick would cause the cursor to traverse the entire screen in a corresponding direction.

When the cursor came into contact with one of the blue walls, a collision was recorded, a tone was presented, and a pellet was dispensed. Because the springs were removed, the joystick did not automatically center itself when released. Thus, subsequent trials would begin with the cursor located in the same position as that at which it had ended on the previous trial. If this was a location normally occupied by a wall, that rectangle would not be displayed on the screen (i.e., no trial began with the cursor already in contact with a blue wall).

The number and size of the walls were also determined by performance. If the mean response time (RT; interval from the first movement of the joystick until contact with a target was recorded) for five consecutive trials was less than $3 \mathrm{sec}$, the number or size of the rectangles was reduced (four rectangles or walls to three walls, to two walls, to one wall, to one half-sized wall, to one quarter-sized wall). If mean RT exceeded $20 \mathrm{sec}$, the number or size of the walls was increased. Each trial was separated by a blank screen for $5 \mathrm{sec}$.

In a second task (chase), the cursor and the smallest single wall (the target stimulus; a blue rectangle, $2.5 \times 1.25 \mathrm{~cm}$ ) from the previous task were used. When the joystick was manipulated in this task, however, both the cursor and the target moved on the screen. The cursor again moved in a direction and to a position isomorphic to the angle of joystick deflection. The target moved diagonally across the screen at $2.5 \mathrm{~cm} / \mathrm{sec}$, "bouncing" off the borders of the $23 \times 14 \mathrm{~cm}$ viewing area at $45^{\circ}$ angles. The initial location and direction of movement for the target was randomly determined each trial, with the constraint that no trial began with the cursor atop the target. On the chase task, bringing the cursor into contact with the moving target stimulus resulted in the same auditory feedback as that used with the side task and, on an average of one third of the trials, in delivery of a $97-\mathrm{mg}$ pellet. This variable ratio (VR) schedule was introduced to increase the number of trials performed by the rat each day.

In the third task (choice), a nontarget stimulus was also added to the chase screen. This stimulus (four concentric circles, the largest of which was $1.25 \mathrm{~cm}$ in radius) produced a $5-\mathrm{sec} 50-\mathrm{Hz}$ buzz and no pellet when touched by the cursor. The nontarget stimulus was randomly positioned at the start of each trial (not overlapping with the cursor or the target) and moved as did the target stimulus. As with the target stimulus, bringing the cursor within $1 \mathrm{~cm}$ of the center of the nontarget was recorded as a response. Responses to the target stimulus were always reinforced with a pellet and with the tone from the side task.

Procedure. Irrespective of the task, the rat was tested in its home cage, which was modified as described above, with the computer task available $24 \mathrm{~h} /$ day. The tasks were introduced in the order described 


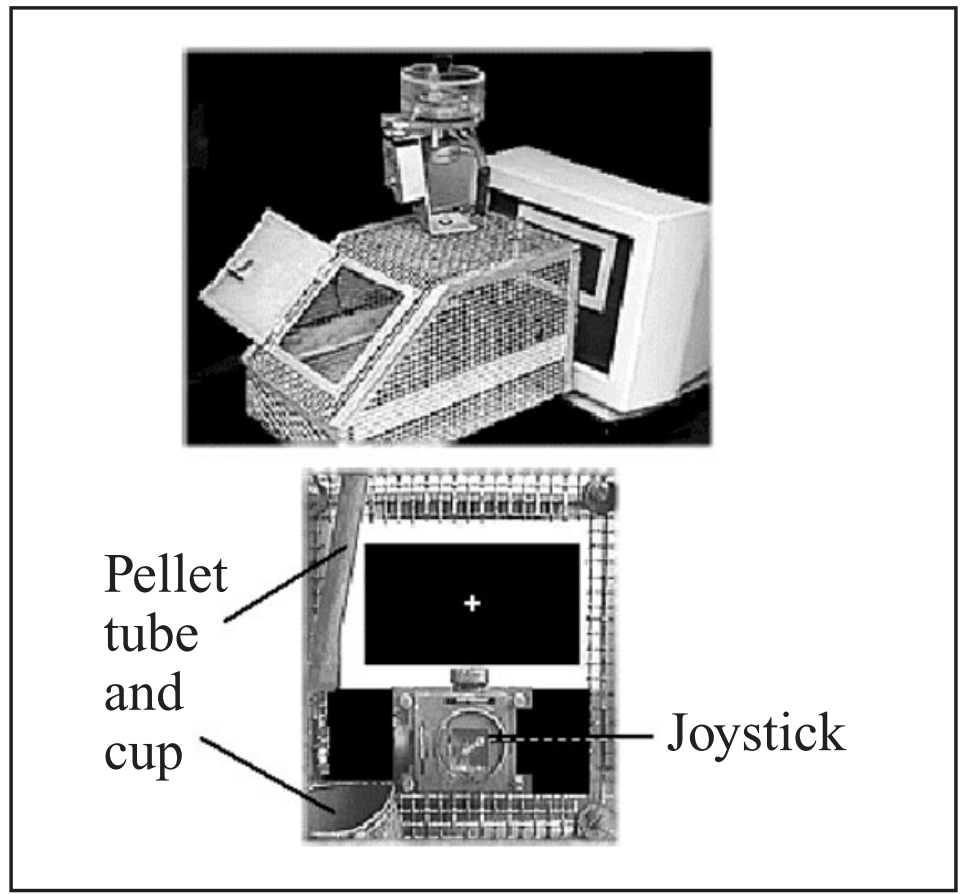

Figure 1. The cage and test system used in this study (top), with a close-up of the screen and joystick as viewed from within the cage (bottom).

above (side, chase, choice), with a new task presented to the animal only after the experimenters concluded that asymptotic performance had been achieved on the previous task in the sequence.

\section{Results}

Side task. No manual shaping was used in this experiment. However, the side task automatically reinforced any large spontaneous movements of the joystick. In this way, the rat learned to move the joystick (using its mouth and paws together to grasp the metal handle) to receive pellets. Figure 2 shows the number of side trials completed successfully across days of the experiment.

On average, the rat performed 326 side trials per day. It should be noted that moving the joystick around persistently will eventually result in the cursor's touching a wall - even if one does not look at the screen. It was dif-

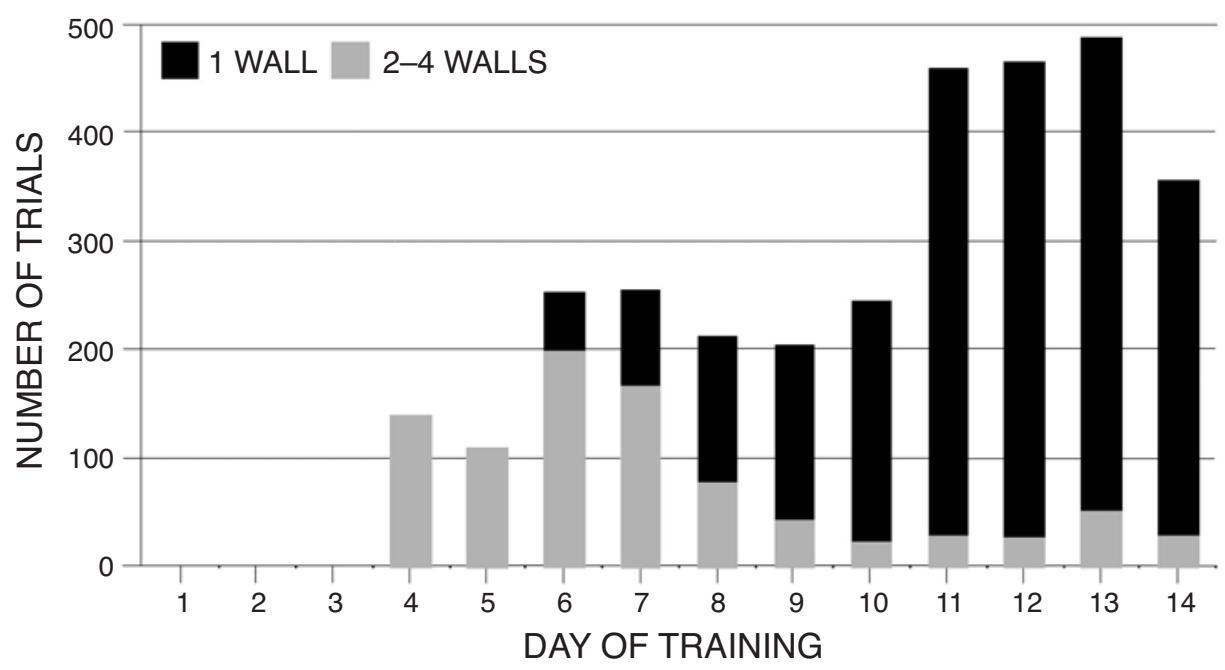

Figure 2. Number of trials per training day as a function of the number of sidewalls available (first 14 days of training). 
ficult to rule out this possibility in the present case, although the cursor tended initially to move in the direction of a target wall even in the one-wall condition $(55 \%$ of the one-wall trials, chance $=.33 ; z=4.67, p<.05)$. However, mean RTs were very high $(12.11 \mathrm{sec})$, reflecting a difficulty in distinguishing the first real movement of the joystick from noise produced by the joystick-reading software and the removal of the joystick springs. This noise caused the cursor to jitter on the screen, bouncing back and forth in an area of several pixels. Critically, this noise could not cause the cursor to drift or contact a target wallonly to obscure the moment at which the rat actually began to manipulate the joystick during each trial.

Chase task. Another way of determining whether the rat was skillfully controlling the cursor or just wiggling the joystick persistently but randomly was to test the animal with a moving stimulus. To increase the number of trials the animal could produce each day without sating, chase trials were reinforced on a VR-3 schedule. Subsequently, the rat increased its productivity to an average of 344 trials/ day. Over 10,000 chase trials were produced, with a mean RT over the last 1,000 trials of $14.96 \mathrm{sec}$.

Choice task. The final test of whether the rat was moving the joystick skillfully or randomly was administered by adding a nontarget stimulus to the screen. Random movement of the joystick would cause the cursor to contact the nontarget (circle) stimulus as often as the target (square). Correct responses were always reinforced. Figure 3 shows accuracy levels per test day. Given an average of about 200 trials per day, $57 \%$ accuracy or better was reliably better than chance $(p<.05)$. The accuracy level obtained in the terminal block (71\%) - which appears to represent asymptote for these conditions - represents a highly reliable difference from chance $(z=5.56, p<.01)$.

\section{Discussion}

An albino rat learned to manipulate a joystick so as to bring a cursor into contact with computer-generated stimuli. Although its RTs remained relatively high (albeit confounded by ambiguity about the onset of joystick manipulation) and its choice task accuracy remains far from perfect, its performance was quite impressive for a colorblind animal with limited visual acuity, manipulating a joystick with its mouth and paws and looking at a color display located several centimeters above its head and outside the cage. More pointedly, its performance was significantly better than what could be expected by chance alone. Although interpretation of these data is admittedly constrained by a number of factors, the fact remains that they cannot be explained as random movements of the manipulandum by the animal. That is, the rat was moving the joystick so as to control the cursor (e.g., to bring it into contact with one, but not the other, computer graphic stimulus), albeit without precision. This is the first such demonstration and, as such, introduces a new range of comparative questions that might be asked with rodents.

\section{EXPERIMENT 2}

One of the limitations of the first experiment is that we could not determine the nature of the rat's interaction with the joystick - that is, in some way other than from the movements of the cursor on the screen. Because the animal had continuous access to the joystick, it typically did not produce trials when the experimenters were present. Videotaping produced some record of its responses, but the quality of the tape was substantially reduced by the layers of mesh and the small scale of the apparatus. Experiment 2 was designed to remedy this lack of descriptive data.

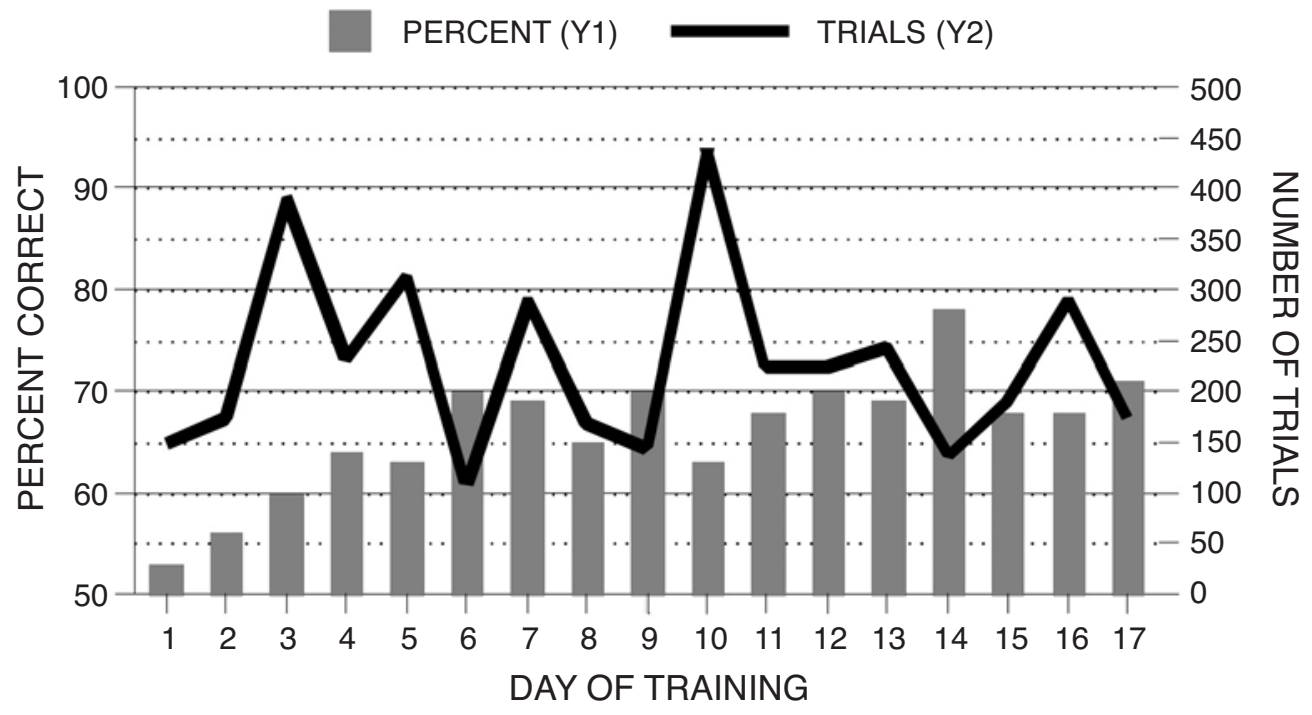

Figure 3. Accuracy on the choice task, by day ( $Y 1$ = left ordinate). The number of trials per day is depicted by the line ( $Y 2=$ right ordinate). 
Of course, it only takes 1 successful rat to demonstrate that rats can succeed in learning this new skill; however, the second experiment was also designed to replicate the findings of Experiment 1. Whereas the animal in the first experiment succeeded on several different tasks designed to show that it was indeed controlling the cursor on the screen in nonrandom ways, the present rats were tested to asymptote on a single joystick task so as to compare their mature performance with that of joystick-competent monkeys.

\section{Method}

Subjects. Two adult female albino rats were tested in this experiment. As in the previous experiment, the animals (weighing between 250 and $260 \mathrm{~g}$ ) were about between 1 and 2 years old when the study began and had received traditional operant leverpressing training prior to the present experiment. Food was initially restricted to maintain the rats at between $90 \%$ and $100 \%$ of their free-feeding weight, after which they able to provide ample food for themselves to remain at their free-feeding weight.

For comparison, 2 rhesus monkeys (Macaca mulatta) were also tested in this experiment. Although the animals were joystick trained and test experienced (see, e.g., Washburn \& Gulledge, 2002) prior to this experiment, the data reported here were the monkeys' first tests with the joystick modified for rodents as described in Experiment 1 . Thus, they provide a baseline of how difficult the present joystick was to control by nonhuman animals.

Apparatus. A second test station like the one built for Experiment 1 was constructed so that both rats could be independently and simultaneously trained. Thus, the rats were tested in their respective home cages, using an apparatus similar to that shown in Figure 1. To accommodate videotaping of the animals, a small portion of the mesh on the right side of each cage was removed and replaced with Lexan. This allowed a camera to be positioned so that an unobstructed view of the joystick could be had.

Procedure. The side task from the previous experiment was again employed to train the animals. The rats were trained and tested by following the side protocol in Experiment 1. The computer automatically titrated the difficulty of each trial, decreasing or increasing the number and the size of the target walls, contingent on each animal's performance. Once a rat had graduated to the most difficult condition, it was allowed to produce at least 1,000 trials in that condition. Performance was examined for each block of 200 trials to determine whether asymptote had been obtained.

Following this testing, the joysticks were removed, cleaned, and installed in the test stations used for the monkeys. Each rhesus monkey in this experiment was then tested on 200 trials of the side task, using the joysticks. Although the task was familiar to each of the monkeys (each monkey had received his own joystick training with the side task), these were the monkeys' first encounters with a joystick that did not return to center when released and with a cursor that moved isomorphically to the position (rather than the direction) of joystick deflection.

\section{Results}

As was observed in Experiment 1, these 2 rats were successfully shaped to manipulate the joystick under increasingly difficult conditions. The side task titrated to the one-wall condition for 1 animal in the 5 th day of training (Trial 605 of training), and the majority of the trials were in the hardest condition within the fifth block of 200 training trials. The other rat progressed somewhat more slowly, reaching the one-wall condition on the 8th day of training (Trial 717). This animal required eight 200-trial training blocks before it was titrating and maintaining the task in the most difficult condition.

By the time that the rats were consistently performing side trials with a quarter-wall-sized target, they were apparently asymptotic in performance. That is, performance did not improve over the next five blocks of 200 trials, whether one was examining the time required to hit the target with the cursor or the accuracy of responding. Because of the sensitivity of the joystick at rest (due to the removal of the springs) and the variable distance that separated the cursor from the target at the outset of each trial, we focused

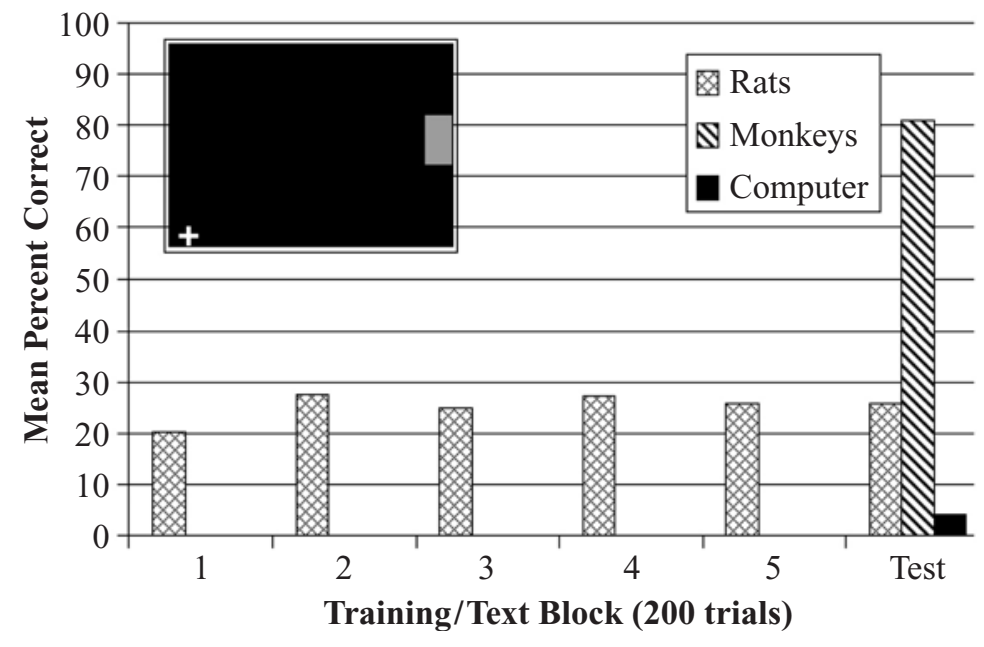

Figure 4. Experiment 2 accuracy (percentage of trials on which the cursor hit the target before it hit any other border of the screen) on the side task by rats, monkeys, and simulation (inset: a possible screen configuration for the test condition). 
on response accuracy for this experiment. An accurate response was defined as moving the cursor into contact with the target before the cursor touched any border of the screen (other than the border against which it had begun). That is, an error was scored if the cursor was brought into contact with any border of the screen, including the side of the screen on which the target was positioned, before hitting the target. Thus, if the target appeared in the top center of the screen with the cursor positioned in the lower right corner (see Figure 4 inset), moving the cursor directly up to the top wall would be scored as an error, just as would wiggling the joystick randomly until the cursor left and then returned to the bottom wall. Only initial contacts with the blue rectangular target were scored as correct responses.

The histogram in Figure 4 shows the rats' mean accuracy on the last 1,200 trials in the most difficult side task condition. There is no evidence of continued improvement in performance across blocks of trials, and each rat was accurate on about one fourth of its first responses. Although the target occupied one fourth of a border of the screen, chance was much less than the $25 \%$ accuracy averaged by the rats, because the target could appear on any of the screen borders not occupied by the cursor at trial outset.

To get an estimate of chance, a computer simulation was written in which the target was positioned and the cursor was moved as in the rats' task, but in which the direction of cursor movement was randomized. As is shown in Figure 4 , this simulation indicated that only about $10 \%$ of the trials would be correct by chance alone. The accuracy levels observed for the rats was significantly in excess of this value $(z=2.89, p<.01)$.

Figure 4 also shows the data for 2 rhesus monkeys, each tested for 200 trials. The monkeys adapted to the new joystick from the first trial, successfully directing the cursor into contact with the target. Their mean accuracy level for the first 200 trials was significantly better than that observed for the rats $(z=9.34, p<.01)$. Indeed, most of the monkeys' errors occurred because the cursor collided with the wall on which the target was positioned, missing the target itself by a few centimeters.

\section{GENERAL DISCUSSION}

In two experiments, rats were shown to learn to control the movements of a computer graphic cursor by manipulating a joystick. An examination of the videotape from Experiment 2 revealed that both rats used a combination of paws and mouth to manipulate the joystick handle. This was true in mature performance, as well as in initial training. This provides one potential reason why this rat's asymptotic levels of performance were so inferior to those observed for monkeys: It is difficult visually to observe events on the computer screen if one also uses the mouth (and thus moves the head) to manipulate a joystick. Given the manual dexterity that rats demonstrate in leverpressing and other contexts, it seems reasonable to anticipate that the animals could learn to manipulate the joystick exclu- sively with the forelimbs, but it remains for further research to demonstrate this intuition empirically.

It is likely that the animals' prior operant training facilitated the present study, in that the animals were both magazine trained and generally experienced at pressing a lever to obtain reinforcers. However, it should be stressed that virtually every aspect of the present training was different from this prior experience. The physical cage and location of the apparatus, the appearance and the movements of the joystick, the use of continuous visual stimuli that moved in accordance with joystick movements, the auditory feedback, and the demands of each of the tasks were novel to the present study. Clearly, in all but the earliest stages of training, it cannot be argued that the present animals were simply leverpressing with the joystick.

It is intriguing to consider the ways that behavioral research with rodents might be changed by this paradigm. However, several problems must be addressed for the computerized test system to produce the exciting scientific advances characteristic of its application to primate research. Notwithstanding, the present data indicate that rodents have the capacity to learn at least some of the tasks that have been developed for monkeys. Already, it seems clear that this testing paradigm will result in high levels of productivity and new opportunities for comparing task performance by rats, monkeys, apes, and humans.

\section{REFERENCES}

Andrews, M. W. (1993). Video-task paradigm extended to Saimiri. Perceptual \& Motor Skills, 76, 183-191.

Astur, R. S., Koerner, A., Barrington, F. M., McDonald, R. J., GonZales, A. Y., O'Brien, C., \& Sutherland, R. J. (1996). The role of the amygdala in conditioned fear and remembering reward size. Society for Neuroscience Abstracts, 22.

Bussey, T. J., Dias, R., Amin, E., Muir, J. L., \& Aggleton, J. P. (2001). Perirhinal cortex and place-object conditional learning in the rat. $\mathrm{Be}$ havioral Neuroscience, 115, 776-785.

Filion, C. M., Washburn, D. A., \& Gulledge, J. P. (1996). Can monkeys (Macaca mulatta) represent invisible displacement? Journal of Comparative Psychology, 110, 386-395.

Heyes, C. M., \& Dawson, G. R. (1990). A demonstration of observational learning in rats using a bidirectional control. Quarterly Journal of Experimental Psychology, 42B, 59-71.

Markham, M. R., Butt, A. E., \& Dougher, M. J. (1996). A computer touch-screen apparatus for training visual discriminations in rats. Journal of the Experimental Analysis of Behavior, 65, 173-182.

MatzKe, S. M. (1999). Effects of global cerebral ischemia on long-term object memory in the rat. Dissertation Abstracts International, 60B, 2393.

Mitchell, C. J., Heyes, C. M., Gardner, M. R., \& Dawson, G. R. (1999). Limitations of a bidirectional control procedure for the investigation of imitation in rats: Odour cues on the manipulandum. Quarterly Journal of Experimental Psychology, 52B, 193-202.

Ray, E. D., Gardner, M. R., \& Heyes, C. M. (2000). Seeing how it's done: Matching conditions for observer rats (Rattus norvegicus) in the bidirectional control. Animal Cognition, 3, 147-157.

Rumbaugh, D. M., Richardson, W. K., Washburn, D. A., SavageRumbaugh, E. S., \& Hopkins, W. D. (1989). Rhesus monkeys (Macaca mulatta), video tasks, and implications for stimulus-response spatial contiguity. Journal of Comparative Psychology, 103, 32-38. Smith, J. D., Shields, W. E., Schull, J., \& Washburn, D. A. (1997) The uncertain response in humans and animals. Cognition, 62, 75-97. VAuclair, J., FAGOT, J., \& Hopkins, W. D. (1993). Rotation of mental 
images in baboons when the visual input is directed to the left cerebral hemisphere. Psychological Science, 4, 99-103.

Washburn, D. A., \& Gulledge, J. P. (2002). A species difference in visuospatial memory in adult humans and rhesus monkeys: The Concentration game. International Journal of Comparative Psychology, 15, 287-301.

Washburn, D. A., Hopkins, W. D., \& Rumbaugh, D. M. (1990). The effects of competition upon video-task performance in monkeys (Macaca mulatta). Journal of Comparative Psychology, 104, 115-121.

WASHBURN, D. A., \& RUMBAUGH, D. M. (1991a). Impaired performance from brief social isolation of rhesus monkeys: A multiple video-task assessment. Journal of Comparative Psychology, 105, 145-151.

Washburn, D. A., \& Rumbaugh, D. M. (1991b). Rhesus monkey (Macaca mulatta) complex learning skills reassessed. International Journal of Primatology, 12, 377-388.
Washburn, D. A., \& Rumbaugh, D. M. (1992a). A comparative assessment of psychomotor performance: Target prediction by humans and macaques. Journal of Experimental Psychology: General, 121, 305-312.

WASHBURn, D. A., \& Rumbaugh, D. M. (1992b). Investigations of rhesus monkeys video-task performance: Evidence for enrichment. Contemporary Topics in Laboratory Animal Science, 31, 6-10.

Washburn, D. A., \& Rumbaugh, D. M. (1992c). Testing primates with joystick-based automated apparatus: Lessons from the Language Research Center's Computerized Test System. Behavior Research Methods, Instruments, \& Computers, 24, 157-164.

(Manuscript received January 13, 2004; accepted for publication March 14, 2004.) 